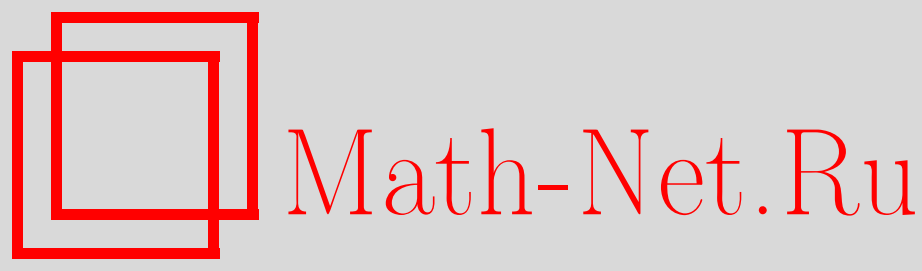

М. М. Рахматуллаев, Слабо периодические меры Гиббса и основные состояния для модели Поттса с конкурирующими взаимодействиями на дереве Кэли, ТМФ, 2013, том 176, номер 3, 477-493

DOI: https://doi.org/10.4213/tmf8530

Использование Общероссийского математического портала Math-Net.Ru подразумевает, что вы прочитали и согласны с пользовательским соглашением http://www.mathnet.ru/rus/agreement

Параметры загрузки:

IP : 3.85 .5 .30

26 апреля 2023 г., 16:34:02

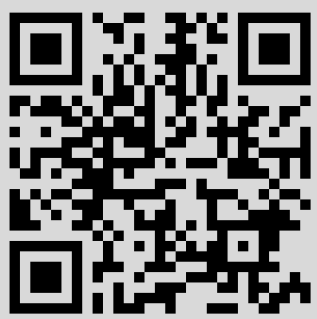


Том 176, № 3

сентябрь, 2013

\title{
СЛАБО ПЕРИОДИЧЕСКИЕ МЕРЫ ГИББСА И ОСНОВНЫЕ СОСТОЯНИЯ ДЛЯ МОДЕЛИ ПОТТСА С КОНКУРИРУЮЩИМИ ВЗАИМОДЕЙСТВИЯМИ НА ДЕРЕВЕ КЭЛИ
}

\begin{abstract}
Для модели Поттса с конкурирующими взаимодействиями на дереве Кэли описано множество слабо периодических основных состояний, соответствующих нормальным делителям индекса 2 группового представления дерева Кэли. Также изучены некоторые слабо периодические меры Гиббса.
\end{abstract}

Ключевые слова: дерево Кэли, мера Гиббса, модель Поттса, слабо периодические основные состояния.

DOI: $1010.4231 / \operatorname{tmf} 8530$

\section{1. ВВЕДЕНИЕ}

Известно, что фазовая диаграмма мер Гиббса для данного гамильтониана близка к фазовой диаграмме основных изолированных (устойчивых) состояний этого гамильтониана. При низких температурах периодическому основному состоянию соответствует периодическая мера Гиббса [1], [2]. В результате возникает задача описания периодических и слабо периодических основных состояний.

Слабо периодические основные состояния для модели Изинга с конкурирующими взаимодействиями описаны в работах [3], [4]. Слабо периодические меры Гиббса изучены в работах [3], [5]. Периодические основные состояния для модели Поттса с конкурирующими взаимодействиями рассмотрены в работе [6].

Дерево Кэли $\tau^{k}$ порядка $k \geqslant 1$ - это бесконечное дерево, т. е. граф без циклов, из каждой вершины которого выходит ровно $k+1$ ребер.

Пусть $\tau^{k}=(V, L, i)$, где $V$ - множество вершин графа $\tau^{k}, L$ - множество его ребер и $i-$ функция инцидентности, сопоставляющая каждому ребру $l \in L$ его концевые точки $x, y \in V$. Если $i(l)=\{x, y\}$, то $x$ и $y$ мы называем ближайшими соседями и обозначаем как $l=\langle x, y\rangle$. Определим расстояние $d(x, y)$ между вершинами $x, y \in V$ на дереве Кэли как минимальное число $d$ такое, что найдутся

*Наманганский государственный университет, Наманган, Узбекистан.

E-mail: mrahmatullaev@rambler.ru 
$x=x_{0}, x_{1}, \ldots, x_{d-1}, x_{d}=y \in V$, которые последовательно являются ближайшими соседями: $\left\langle x_{0}, x_{1}\right\rangle, \ldots,\left\langle x_{d-1}, x_{d}\right\rangle$. Для фиксированного $x^{0} \in V$ введем обозначения

$$
\begin{aligned}
W_{n} & =\left\{x \in V: d\left(x, x^{0}\right)=n\right\}, \\
V_{n} & =\left\{x \in V: d\left(x, x^{0}\right) \leqslant n\right\}, \\
L_{n} & =\left\{l=\langle x, y\rangle \in L: x, y \in V_{n}\right\} .
\end{aligned}
$$

Для $x \in W_{n}$ назовем множеством прямых потомков множество

$$
S(x)=\left\{y \in W_{n+1}: d(x, y)=1\right\} .
$$

Мы рассматриваем модели, в которых спин принимает значения из множества $\Phi=\{1,2, \ldots, q\}, q \geqslant 2$. Конфигурация $\sigma$ на множестве вершин $V$ определяется как функция $x \in V \rightarrow \sigma(x) \in \Phi$; множество всех конфигураций совпадает с $\Omega=\Phi^{V}$.

Известно, что дерево Кэли $\tau^{k}$ можно представить как свободное произведение $G_{k}$ $k+1$ циклических групп второго порядка (см., например, работы [3]-[9]). Обозначим через $S_{1}(x)$ множество всех ближайших соседей точки $x \in G_{k}$, т. е.

$$
S_{1}(x)=\left\{y \in G_{k}:\langle x, y\rangle\right\} .
$$

Пусть $x_{\downarrow}=S_{1}(x) \backslash S(x)$.

Определим $G_{k}^{*}$-периодическую конфигурацию $\sigma(x)$, которая является инвариантной относительно подгруппы $G_{k}^{*} \subset G_{k}$ конечного индекса, т. е. $\sigma(y x)=\sigma(x)$ для любых $x \in G_{k}, y \in G_{k}^{*}$. Для данной периодической конфигурации индекс подгруппы называется периодом конфигурации. Пусть $G_{k} / G_{k}^{*}=\left\{H_{1}, \ldots, H_{r}\right\}$ - факторгруппа, где $G_{k}^{*}-$ нормальный делитель индекса $r \geqslant 1$. Конфигурацию $\{\sigma(x), x \in V\}$ назовем $G_{k}^{*}$-слабо периодической, если $\sigma(x)=\sigma_{i j}$ при $x_{\downarrow} \in H_{i}, x \in H_{j}$ для любого $x \in G_{k}$, другими словами, значение конфигурации зависит не от $x$, а от номеров классов принадлежности вершин $x$ и $x_{\downarrow}$.

Гамильтониан модели Поттса с конкурирющими взаимодействиями имеет вид

$$
H(\sigma)=J_{1} \sum_{\substack{x, y \in V,\langle x, y\rangle}} \delta_{\sigma(x) \sigma(y)}+J_{2} \sum_{\substack{x, y \in V: \\ d(x, y)=2}} \delta_{\sigma(x) \sigma(y)},
$$

где $J_{1}, J_{2} \in \mathbb{R}, \delta_{u v}$ - символ Кронекера.

В настоящей работе рассматривается модель Поттса с конкурирующими взаимодействиями. Цель работы - описать слабо периодические основные состояния для этой модели и слабо периодические меры Гиббса для обычной модели Поттса $\left(J_{2}=0\right)$. В разделе 2 сформулированы результаты о слабо периодических основных состояниях. В разделе 3 приведены результаты о слабо периодических мерах Гиббса. Доказательства всех результатов даны в разделе 4.

\section{2. ОСНОВНЫЕ СОСТОЯНИЯ}

Для пары конфигураций $\sigma$ и $\varphi$, совпадающих почти всюду, т. е. всюду, за исключением конечного числа точек, мы рассматриваем относительный гамильтониан $H(\sigma, \varphi)$, определяющий различие между энергиями конфигураций $\sigma$ и $\varphi$ :

$$
H(\sigma, \varphi)=J_{1} \sum_{\substack{x, y \in V,\langle x, y\rangle}}\left(\delta_{\sigma(x) \sigma(y)}-\delta_{\varphi(x) \varphi(y)}\right)+J_{2} \sum_{\substack{x, y \in V, d(x, y)=2}}\left(\delta_{\sigma(x) \sigma(y)}-\delta_{\varphi(x) \varphi(y)}\right),
$$

где $J=\left(J_{1}, J_{2}\right) \in \mathbb{R}^{2}$ - произвольный фиксированный параметр. 
Пусть $M$ - множество единичных шаров с вершинами в $V$. Назовем ограниченной конфигуращией $\sigma_{b}$ сужение конфигурации $\sigma$ на шар $b \in M$. Определим энергию конфигурации $\sigma_{b}$ на $b$ следующим образом:

$$
U\left(\sigma_{b}\right) \equiv U\left(\sigma_{b}, J\right)=\frac{J_{1}}{2} \sum_{\substack{x, y \in b,\langle x, y\rangle}} \delta_{\sigma(x) \sigma(y)}+J_{2} \sum_{\substack{x, y \in b, d(x, y)=2}} \delta_{\sigma(x) \sigma(y)},
$$

где $J=\left(J_{1}, J_{2}\right) \in \mathbb{R}^{2}$.

Известна следующая лемма [3], [6].

ЛЕмма 1. Относительный гамильтониан (1) имеет вид

$$
H(\sigma, \varphi)=\sum_{b \in M}\left(U\left(\sigma_{b}\right)-U\left(\varphi_{b}\right)\right)
$$

В этом разделе мы рассмотрим случай $q=3$, т. е. $|\Phi|=3$. Пусть $e$ - единичный элемент из группы $G_{k}$. Обозначим через $c_{b}$ центр единичного шара $b$, положим $B_{t}=\left\{x \in S_{1}(e): \varphi_{b}(x)=t\right\}$ для любого $t \in \Phi$. Пусть $\varphi_{b}\left(c_{b}\right)=d,\left|B_{d}\right|=i,\left|B_{f}\right|=n$, тогда $\left|B_{g}\right|=k+1-i-n$, где $d \neq f, d \neq g, f \neq g, d, f, g \in \Phi$. Легко доказать следующую лемму.

ЛЕмма 2. Для каждой конфигурации $\varphi_{b}$ имеет место следующее включение:

$$
U\left(\varphi_{b}\right) \in\left\{U_{i, n}: i=0,1, \ldots, k+1, n=0,1, \ldots,\left[\frac{k+1-i}{2}\right]\right\},
$$

¿de

$$
U_{i, n}=\frac{J_{1}}{2} i+\frac{J_{2}}{2}(i(i-1)+(k-n-i)(k-n-i+1)+n(n-1))
$$

$u[\cdot]$ - челая часть числа.

ОПРЕДЕЛЕНИЕ 1. Конфигурация $\varphi$ называется основным состоянием относительного гамильтониана $H$, если

$$
U\left(\varphi_{b}\right)=\min \left\{U_{i, n}: i=0,1, \ldots, k+1, n=0,1, \ldots,\left[\frac{k+1-i}{2}\right]\right\}
$$

для любого $b \in M$.

$$
\begin{aligned}
& \text { Положим } C_{i, n}=\left\{\varphi_{b}: U\left(\varphi_{b}\right)=U_{i, n}\right\} \text { и } \\
& A_{\xi, \eta}=\left\{J \in \mathbb{R}^{2}: U_{\xi, \eta}=\min \left\{U_{i, n}: i=0,1, \ldots, k+1, n=0,1, \ldots,\left[\frac{k+1-i}{2}\right]\right\}\right\} .
\end{aligned}
$$

2.1. Случай $k=2$. Легко видеть, что в этом случае для любой конфигурации $\sigma_{b}$

$$
U\left(\sigma_{b}\right) \in\left\{U_{0,0}, U_{0,1}, U_{1,0}, U_{1,1}, U_{2,0}, U_{3,0}\right\}
$$

где

$$
\begin{aligned}
U_{0,0} & =3 J_{2}, & U_{0,1} & =J_{2}, \\
U_{1,1} & =\frac{1}{2} J_{1}, & U_{1,0} & =\frac{1}{2} J_{1}+J_{2}, \\
U_{2,0} & =J_{1}+J_{2}, & U_{3,0} & =\frac{3}{2} J_{1}+3 J_{2} .
\end{aligned}
$$


Используя определение (3), имеем

$$
\begin{aligned}
& A_{0,0}=\left\{J \in \mathbb{R}^{2}: J_{1} \geqslant 0, J_{2} \leqslant 0\right\}, \\
& A_{0,1}=\left\{J \in \mathbb{R}^{2}: J_{2} \geqslant 0, J_{1}-2 J_{2} \geqslant 0\right\}, \\
& A_{1,0}=\left\{J \in \mathbb{R}^{2}: J_{1}=0, J_{2}=0\right\}, \\
& A_{1,1}=\left\{J \in \mathbb{R}^{2}: J_{2} \geqslant 0,-2 J_{2} \leqslant J_{1} \leqslant 2 J_{2}\right\}, \\
& A_{2,0}=\left\{J \in \mathbb{R}^{2}: J_{2} \geqslant 0,-4 J_{2} \leqslant J_{1} \leqslant-2 J_{2}\right\}, \\
& A_{3,0}=\left\{J \in \mathbb{R}^{2}: J_{1} \leqslant 0, J_{1}+4 J_{2} \leqslant 0\right\}
\end{aligned}
$$

и $\bigcup_{i, n} A_{i, n}=\mathbb{R}^{2}$.

Пусть $A \subset\{1,2, \ldots, k+1\}$. Положим

$$
H_{A}=\left\{x \in G_{k}: \sum_{j \in A} w_{j}(x)-\text { четное число }\right\} \text {, }
$$

где $w_{j}(x)$ - количество символов $a_{j}$ в слове $x$. Множество $H_{A}$ является нормальным делителем индекса 2.

Рассмотрим факторгруппу $G_{k} / H_{A}=\left\{H_{A}, G_{k} \backslash H_{A}\right\}$. Для простоты введем обозначения $H_{0}=H_{A}, H_{1}=G_{k} \backslash H_{A}$. $H_{A}$-слабо периодические конфигурации имеют следующий вид:

$$
\varphi(x)= \begin{cases}a_{00}, & \text { если } x_{\downarrow} \in H_{0}, x \in H_{0}, \\ a_{01}, & \text { если } x_{\downarrow} \in H_{0}, x \in H_{1}, \\ a_{10}, & \text { если } x_{\downarrow} \in H_{1}, x \in H_{0}, \\ a_{11}, & \text { если } x_{\downarrow} \in H_{1}, x \in H_{1},\end{cases}
$$

где $a_{i j} \in \Phi, i, j \in\{0,1\}$.

Теорема 1. Пусть $J_{1} \neq 0, J_{2} \neq 0,|A|=1$. При $k=2, q=3$ все $H_{A}$-слабо периодические основные состояния являются периодическими или трансляционноинвариантными.

ТеОрема 2. Пусть $|A|=2$. При $k=2, q=3$ верны следующие утверждения.

1. На множестве $A_{0,1}$ существуют шесть $H_{A}$-слабо периодических основных состояний, не являющихся периодическими или трансляционно-инвариантными; они имеют вид

$$
\varphi^{*}(x)= \begin{cases}l, & \text { если } x_{\downarrow} \in H_{0}, x \in H_{0}, \\ m, & \text { если } x_{\downarrow} \in H_{0}, x \in H_{1}, \\ n, & \text { если } x_{\downarrow} \in H_{1}, x \in H_{0}, \\ l, & \text { если } x_{\downarrow} \in H_{1}, x \in H_{1},\end{cases}
$$

где $l, m, n \in \Phi$ u $l \neq m, l \neq n, m \neq n$.

2. Любые $H_{A}$-слабо периодические основные состояния, кроме $\varphi^{*}(x)$, являются трансляционно-инвариантными.

ЗАмЕчАниЕ 1. Известно, что периодические и трансляционно-инвариантные основные состояния являются также слабо периодическими, но обратное неверно. Поэтому найденные основные состояния являются новыми. 
2.2. Случай $k=3$. При $k=3$ из леммы 2 для любого $\sigma_{b}$ получим

$$
U\left(\sigma_{b}\right) \in\left\{U_{0,0}, U_{0,1}, U_{0,2}, U_{1,0}, U_{1,1}, U_{2,0}, U_{2,1}, U_{3,0}, U_{4,0}\right\}
$$

где

$$
\begin{aligned}
& U_{0,0}=6 J_{2}, \quad U_{0,1}=3 J_{2}, \quad U_{0,2}=2 J_{2}, \quad U_{1,0}=\frac{1}{2} J_{1}+3 J_{2}, \\
& U_{1,1}=\frac{1}{2} J_{1}+J_{2}, \quad U_{2,0}=J_{1}+2 J_{2}, \quad U_{2,1}=J_{1}+J_{2}, \quad U_{3,0}=\frac{3}{2} J_{1}+3 J_{2}, \\
& U_{4,0}=2 J_{1}+6 J_{2} \text {. }
\end{aligned}
$$

Воспользуемся определением (3). Нетрудный, но достаточно громоздкий анализ показывает, что

$$
\begin{aligned}
& A_{0,0}=\left\{J \in \mathbb{R}^{2}: J_{1} \geqslant 0, J_{2} \leqslant 0\right\}, \\
& A_{0,1}=\left\{J \in \mathbb{R}^{2}: J_{1} \geqslant 0, J_{2}=0\right\}, \\
& A_{0,2}=\left\{J \in \mathbb{R}^{2}: 0 \leqslant J_{2} \leqslant J_{1} / 2\right\}, \\
& A_{1,0}=\left\{J \in \mathbb{R}^{2}: J_{1}=0, J_{2}=0\right\}, \\
& A_{1,1}=\left\{J \in \mathbb{R}^{2}: J_{1} \geqslant 0, J_{1}-2 J_{2} \leqslant 0\right\}, \\
& A_{2,0}=\left\{J \in \mathbb{R}^{2}: J_{1}=0, J_{2}=0\right\}, \\
& A_{2,1}=\left\{J \in \mathbb{R}^{2}: J_{1} \leqslant 0, J_{1}+4 J_{2} \geqslant 0\right\}, \\
& A_{3,0}=\left\{J \in \mathbb{R}^{2}: J_{1} \leqslant 0,-J_{1} / 6 \leqslant J_{2} \leqslant-J_{1} / 4\right\}, \\
& A_{4,0}=\left\{J \in \mathbb{R}^{2}: J_{1} \leqslant 0, J_{1}+6 J_{2} \leqslant 0\right\}
\end{aligned}
$$

и $\bigcup_{i, n} A_{i, n}=\mathbb{R}^{2}$. Рассмотрим слабо периодическую конфигурацию (5).

Teоpema 3. Пусть $J_{1} \neq 0, J_{2} \neq 0 u|A|=1,2$. Тогда при $k=3$ u $q=3$ любое $H_{A}$-слабо периодическое основное состояние является периодическим или трансляционно-инвариантным.

Теорема 4. Пусть $|A|=3$. Тогда при $k=3, q=3$ справедливы следующие утверждения.

1. На множестве $A_{0,1}$ существуют шесть $H_{A}$-слабо периодических основных состояний, не являющихся периодическими или трансляционно-инвариантными; они имеют вид (6).

2. Любые $H_{A}$-слабо периодические основные состояния, кроме $\varphi^{*}(x)$, являются трансляиионно-инвариантными.

ЗАмечание 2. В работе [4] доказано, что обычная модель Изинга не имеет слабо периодических (не периодических) основных состояний. Из теоремы 2 и теоремы 4 следует, что обычная модель Поттса (т. е. модель с $J_{2}=0$ ) имеет слабо периодические основные состояния.

2.3. Случай $k \geqslant 4$. В этом пункте мы покажем, что при любом $k \geqslant 4$ существуют слабо периодические (не периодические) основные состояния. 
С помощью определения (2) в некоторых случаях мы можем вычислить $U_{i, n}$ :

$$
\begin{array}{ll}
U_{0,0}=\frac{J_{2}}{2} k(k+1), & U_{0,1}=\frac{J_{2}}{2} k(k-1), \\
U_{0,2}=\frac{J_{2}}{2}((k-2)(k-1)+2), & U_{0,3}=\frac{J_{2}}{2}((k-3)(k-2)+6) .
\end{array}
$$

Используя равенство (3), вычислим $A_{0,1}, A_{0,2}$. Сначала найдем $A_{0,1}$. Для этого заметим, что из неравенства $U_{0,1} \leqslant U_{0,0}$ следует, что $J_{2} \geqslant 0$, а из неравенства $U_{0,1} \leqslant U_{0,2}$ следует, что $J_{2} \leqslant 0$, отсюда $J_{2}=0$. Далее, при $i>0$ из неравенства $U_{0,1} \leqslant U_{i, n}$ следует, что $J_{1} \geqslant 0$. Таким образом, получаем

$$
A_{0,1}=\left\{J \in \mathbb{R}^{2}: J_{1} \geqslant 0, J_{2}=0\right\} .
$$

Аналогичным образом можно вычислить $A_{0,2}$, в результате получаем

$$
A_{0,2}=\left\{J \in \mathbb{R}^{2}: J_{1} \geqslant 0, J_{2}=0\right\} .
$$

Имеет место следующее утверждение.

ТЕОРема 5. При $k \geqslant 4 u|A|=k$ слабо периодическая конфигурация (6) на множестве $\left\{J \in \mathbb{R}^{2}: J_{1} \geqslant 0, J_{2}=0\right\}$ является слабо периодическим основным состоянием.

\section{3. СЛАБО ПЕРИОДИЧЕСКИЕ МЕРЫ}

Гамильтониан обычной модели Поттса с внешним полем $\alpha$ определяется как

$$
H(\sigma)=-J \sum_{\langle x, y\rangle \in L} \delta_{\sigma(x) \sigma(y)}-\alpha \sum_{x \in V} \delta_{1 \sigma(x)},
$$

где $J, \alpha \in \mathbb{R}$.

Определим конечномерное распределение вероятностной меры $\mu$ в обьеме $V_{n}$ :

$$
\mu_{n}\left(\sigma_{n}\right)=Z_{n}^{-1} \exp \left\{-\beta H_{n}\left(\sigma_{n}\right)+\sum_{x \in W_{n}} h_{\sigma(x), x}\right\},
$$

где $\beta=1 / T, T$ - температура, $T>0, Z_{n}^{-1}-$ нормировочный множитель,

$$
\left\{h_{x}=\left(h_{1, x}, h_{2, x}, \ldots, h_{q, x}\right) \in \mathbb{R}^{q}, x \in V\right\}
$$

- совокупность векторов и

$$
H_{n}\left(\sigma_{n}\right)=-J \sum_{\langle x, y\rangle \in L_{n}} \delta_{\sigma(x) \sigma(y)}-\alpha \sum_{x \in V_{n}} \delta_{1 \sigma(x)} .
$$

Говорят, что вероятностное распределение (8) согласованное, если для всех $n \geqslant 1$ и $\sigma_{n-1} \in \Phi^{V_{n-1}}$

$$
\sum_{\omega_{n} \in \Phi^{W_{n}}} \mu_{n}\left(\sigma_{n-1} \vee \omega_{n}\right)=\mu_{n-1}\left(\sigma_{n-1}\right) .
$$


Здесь $\sigma_{n-1} \vee \omega_{n}$ есть объединение конфигураций. В этом случае существует единственная мера $\mu$ на $\Phi^{V}$ такая, что для всех $n$ и $\sigma_{n} \in \Phi^{V_{n}}$

$$
\mu\left(\left\{\left.\sigma\right|_{V_{n}}=\sigma_{n}\right\}\right)=\mu_{n}\left(\sigma_{n}\right) .
$$

Эта мера называется расщепленной мерой Гиббса, соответствующей гамильтониану (7) и векторнозначной функции $h_{x}, x \in V$.

Следующее утверждение [7] описывает условие на $h_{x}$, обеспечивающее согласованность мер $\mu_{n}\left(\sigma_{n}\right)$.

Теорема 6. Вероятностное распределение $\mu_{n}\left(\sigma_{n}\right), n=1,2, \ldots$, заданное в (8), является согласованной мерой тогда и только тогда, когда для любого $x \in V$ имеет место следующее равенство:

$$
h_{x}=\sum_{y \in S(x)} F\left(h_{y}, \theta, \alpha\right),
$$

где $S(x)$ - множество прямых потомков точки $x$ и функиия

$$
F: h=\left(h_{1}, h_{2}, \ldots, h_{q-1}\right) \in \mathbb{R}^{q-1} \rightarrow F(h, \theta, \alpha)=\left(F_{1}, F_{2}, \ldots, F_{q-1}\right) \in \mathbb{R}^{q-1}
$$

определяется как

$$
F_{i}=\alpha \beta \delta_{1 i}+\ln \frac{(\theta-1) e^{h_{i}}+\sum_{j=1}^{q-1} e^{h_{j}}+1}{\theta+\sum_{j=1}^{q-1} e^{h_{j}}}, \quad \theta=e^{J \beta} .
$$

Пусть $G_{k} / G_{k}^{*}=\left\{H_{1}, H_{2}, \ldots, H_{r}\right\}$ - факторгруппа, где $G_{k}^{*}$ - нормальный делитель индекса $r \geqslant 1$.

ОПРедЕлЕниЕ 2. Совокупность векторов $h=\left\{h_{x}, x \in G_{k}\right\}$ называется $G_{k}^{*}$-nерuодической, если $h_{y x}=h_{x}$ для всех $x \in G_{k}, y \in G_{k}^{*}$. При этом $G_{k}$-периодические совокупности называются трансляиионно-инвариантными.

ОПредЕЛЕниЕ 3 . Совокупность векторов $h=\left\{h_{x}, x \in G_{k}\right\}$ называется $G_{k}^{*}$-слабо периодической, если $h_{x}=h_{i j}$ при $x \in H_{i}, x_{\downarrow} \in H_{j}$ для любого $x \in G_{k}$.

ОПРЕДЕЛЕниЕ 4 . Мера $\mu$ называется $G_{k}^{*}$-периодической (слабо периодической), если она соответствует $G_{k}^{*}$-периодической (слабо периодической) совокупности векторов $h$.

Цель этого раздела - описать слабо периодические меры Гиббса.

Рассмотрим случай $\alpha=0$. Пусть $q$ - произвольное натуральное число, т. е. $\sigma: V \rightarrow \Phi=\{1,2, \ldots, q\}$. Для $A \subset\{1,2, \ldots, k+1\}$ рассмотрим множество (4), пусть $G_{k} / H_{A}=\left\{H_{A}, G_{k} \backslash H_{A}\right\}$ - факторгруппа. Вновь для простоты положим $H_{0}=H_{A}$, $H_{1}=G_{k} \backslash H_{A}$.

$H_{A}$-слабо периодические совокупности векторов $h=\left\{h_{x} \in \mathbb{R}^{q-1}, x \in G_{k}\right\}$ имеют следующий вид:

$$
h_{x}= \begin{cases}h_{1}, & \text { если } x_{\downarrow} \in H_{0}, x \in H_{0}, \\ h_{2}, & \text { если } x_{\downarrow} \in H_{0}, x \in H_{1}, \\ h_{3}, & \text { если } x_{\downarrow} \in H_{1}, x \in H_{0}, \\ h_{4}, & \text { если } x_{\downarrow} \in H_{1}, x \in H_{1} .\end{cases}
$$


Здесь $h_{i}=\left(h_{i 1}, h_{i 2}, \ldots, h_{i, q-1}\right), i=1,2,3,4$. Тогда в силу равенства (9) имеем

$$
\begin{aligned}
& h_{1}=(k-|A|) F\left(h_{1}, \theta\right)+|A| F\left(h_{2}, \theta\right), \\
& h_{2}=(|A|-1) F\left(h_{3}, \theta\right)+(k+1-|A|) F\left(h_{4}, \theta\right), \\
& h_{3}=(|A|-1) F\left(h_{2}, \theta\right)+(k+1-|A|) F\left(h_{1}, \theta\right), \\
& h_{4}=(k-|A|) F\left(h_{4}, \theta\right)+|A| F\left(h_{3}, \theta\right) .
\end{aligned}
$$

При $k=|A|$ эта система уравнений имеет следуюший вид:

$$
\begin{array}{ll}
h_{1}=k F\left(h_{2}, \theta\right), & h_{2}=(k-1) F\left(h_{3}, \theta\right)+F\left(h_{4}, \theta\right), \\
h_{3}=(k-1) F\left(h_{2}, \theta\right)+F\left(h_{1}, \theta\right), & h_{4}=k F\left(h_{3}, \theta\right) .
\end{array}
$$

Введем следующие обозначения: $z_{i j}=e^{h_{i j}}, i=1,2,3,4, j=1,2, \ldots, q-1$. Тогда последнюю систему уравнений можно переписать как

$$
\begin{aligned}
& z_{1 j}=\left(\frac{(\theta-1) z_{2 j}+\sum_{i=1}^{q-1} z_{2 i}+1}{\sum_{i=1}^{q-1} z_{2 i}+\theta}\right)^{k}, \\
& z_{2 j}=\left(\frac{(\theta-1) z_{3 j}+\sum_{i=1}^{q-1} z_{3 i}+1}{\sum_{i=1}^{q-1} z_{3 i}+\theta}\right)^{k-1}\left(\frac{(\theta-1) z_{4 j}+\sum_{i=1}^{q-1} z_{4 i}+1}{\sum_{i=1}^{q-1} z_{4 i}+\theta}\right), \\
& z_{3 j}=\left(\frac{(\theta-1) z_{2 j}+\sum_{i=1}^{q-1} z_{2 i}+1}{\sum_{i=1}^{q-1} z_{2 i}+\theta}\right)^{k-1}\left(\frac{(\theta-1) z_{1 j}+\sum_{i=1}^{q-1} z_{1 i}+1}{\sum_{i=1}^{q-1} z_{1 i}+\theta}\right), \\
& z_{4 j}=\left(\frac{(\theta-1) z_{3 j}+\sum_{i=1}^{q-1} z_{3 i}+1}{\sum_{i=1}^{q-1} z_{3 i}+\theta}\right)^{k} ;
\end{aligned}
$$

здесь $j=1,2, \ldots, q-1$.

Рассмотрим отображение $W: \mathbb{R}^{4 q-4} \rightarrow \mathbb{R}^{4 q-4}$, определенное следующим образом:

$$
\begin{aligned}
& z_{1 j}^{\prime}=\left(\frac{(\theta-1) z_{2 j}+\sum_{i=1}^{q-1} z_{2 i}+1}{\sum_{i=1}^{q-1} z_{2 i}+\theta}\right)^{k}, \\
& z_{2 j}^{\prime}=\left(\frac{(\theta-1) z_{3 j}+\sum_{i=1}^{q-1} z_{3 i}+1}{\sum_{i=1}^{q-1} z_{3 i}+\theta}\right)^{k-1}\left(\frac{(\theta-1) z_{4 j}+\sum_{i=1}^{q-1} z_{4 i}+1}{\sum_{i=1}^{q-1} z_{4 i}+\theta}\right), \\
& z_{3 j}^{\prime}=\left(\frac{(\theta-1) z_{2 j}+\sum_{i=1}^{q-1} z_{2 i}+1}{\sum_{i=1}^{q-1} z_{2 i}+\theta}\right)^{k-1}\left(\frac{(\theta-1) z_{1 j}+\sum_{i=1}^{q-1} z_{1 i}+1}{\sum_{i=1}^{q-1} z_{1 i}+\theta}\right), \\
& z_{4 j}^{\prime}=\left(\frac{(\theta-1) z_{3 j}+\sum_{i=1}^{q-1} z_{3 i}+1}{\sum_{i=1}^{q-1} z_{3 i}+\theta}\right)^{k} ;
\end{aligned}
$$

здесь $j=1,2, \ldots, q-1$. Система (10) эквивалентна уравнению $z=W(z)$.

Отображение $W$ имеет следующие инвариантные множества: множество $I_{1}$ элементов $z \in \mathbb{R}^{4 q-4}$ задается условиями

$$
z_{11}=\cdots=z_{1, q-1}=z_{21}=\cdots=z_{2, q-1}=z_{31}=\cdots=z_{3, q-1}=z_{41}=\cdots=z_{4, q-1},
$$

а множество $I_{2}-$ условиями

$$
z_{11}=\cdots=z_{1, q-1}=z_{41}=\cdots=z_{4, q-1}, \quad z_{21}=\cdots=z_{2, q-1}=z_{31}=\cdots=z_{3, q-1} .
$$


ТеОРема 7. Имеют место следующие утверждения.

1. Для модели Поттса все $H_{A}$-слабо периодические меры Гиббса на $I_{1}$ являются трансляиионно-инвариантными.

2. При $k=2 u|A|=1,2$ существует критическое значение $\theta_{\mathrm{cr}}=1+2 \sqrt{q-1}$ такое, что при $\theta>\theta_{\text {сг }}$ существуют по менъшей мере три $H_{A}$-слабо периодические меры Гиббса; при $\theta=\theta_{\text {сr }}$ существуют по менъшей мере две $H_{A}$-слабо периодические меры Гиббса; при $\theta<\theta_{\text {cr }}$ существует по менъшей мере одна $H_{A}$-слабо периодическая мера Гиббса.

Теперь рассмотрим случай произвольного $k$. Легко проверить, что при любом $k$ на инвариантном множестве $I_{2}$ система уравнений (10) принимает вид

$$
\begin{aligned}
& z_{11}=\left(\frac{(\theta+q-2) z_{21}+1}{(q-1) z_{21}+\theta}\right)^{k} \\
& z_{21}=\left(\frac{(\theta+q-2) z_{21}+1}{(q-1) z_{21}+\theta}\right)^{k-1}\left(\frac{(\theta+q-2) z_{11}+1}{(q-1) z_{11}+\theta}\right) .
\end{aligned}
$$

Введем следующее обозначение:

$$
f(z)=\frac{(\theta+q-2) z+1}{(q-1) z+\theta} .
$$

Тогда систему уравнений (11) можно переписать как

$$
z_{11}^{1 / k}=f\left(z_{11}^{(k-1) / k} f\left(z_{11}\right)\right), \quad z_{21}=\left(f\left(z_{21}\right)\right)^{k-1} f\left(z_{11}\right) .
$$

Положим $z=z_{11}^{1 / k}$. Тогда первое уравнение этой системы уравнений принимает следующий вид:

$$
f\left(z^{k-1} f\left(z^{k}\right)\right)=z .
$$

ЛЕмма 3. Все положительные решения уравнения (13) являются положительными решениями уравнения $f\left(z^{k}\right)=z$.

Теорема 8. При $k=|A|$ для модели Поттса на инвариантном множестве $I_{2}$ существует критическое значение $\theta_{\mathrm{cr}}>1$, такое, что при $\theta=\theta_{\mathrm{cr}}$ существуют по менъшей мере две $H_{A}$-слабо периодические меры Гиббса; если $\theta>\theta_{\mathrm{cr}}$, то существуют по менъшей мере три $H_{A}$-слабо периодические меры Гиббса.

ЗАмЕчАниЕ 3. Полученные в теоремах 7, 8 слабо периодические меры являются также трансляционно-инвариантными. Действительно, из системы уравнений (11) с учетом обозначения (12) получим следующее уравнение:

$$
z_{11} f\left(z_{11}\right)=z_{21} f\left(z_{21}\right) .
$$

Рассмотрим производную функции $\phi(z)=z f(z)$ :

$$
\phi^{\prime}(z)=\frac{(q-1)(\theta+q-2) z^{2}+2 \theta(\theta+q-2) z+\theta}{((q-1) z+\theta)^{2}}>0,
$$

т. е. функция $\phi(z)$ строго возрастает. Отсюда соотношение (14) верно тогда и только тогда, когда $z_{11}=z_{21}$. 


\section{4. ДОКАЗАТЕЛЬСТВА ТЕОРЕМ}

4.1. Доказательство теоремы 1. Если $a_{i j}=p \in \Phi$ для любых $i, j \in\{0,1\}$, то очевидно, что соответствующие конфигурации являются трансляционно-инвариантными.

Рассмотрим случай, когда $a_{i j}$ принимает два разных значения из множества $\Phi$.

1. Пусть $a_{00}=a_{01}=a_{10}=l, a_{11}=m$, где $l, m \in \Phi, l \neq m$.

Пусть $c_{b} \in H_{0}$. Возможны следующие случаи:

a) если $c_{b \downarrow} \in H_{0}$ и $\varphi_{b}\left(c_{b \downarrow}\right)=l$, то $\varphi_{b}\left(c_{b}\right)=l,\left|B_{l}\right|=3,\left|B_{m}\right|=0$, следовательно, $\varphi_{b} \in C_{3,0}$

б) если $c_{b \downarrow} \in H_{1}$ и $\varphi_{b}\left(c_{b \downarrow}\right)=m$, то $\varphi_{b}\left(c_{b}\right)=l,\left|B_{l}\right|=2,\left|B_{m}\right|=1$, следовательно, $\varphi_{b} \in C_{2,0}$.

Пусть $c_{b} \in H_{1}$. Возможен следующий случай: если $c_{b \downarrow} \in H_{0}$ и $\varphi_{b}\left(c_{b \downarrow}\right)=l$, то $\varphi_{b}\left(c_{b}\right)=l,\left|B_{l}\right|=1,\left|B_{m}\right|=2$, следовательно, $\varphi_{b} \in C_{1,0}$; но, поскольку мы имеем $A_{3,0} \cap A_{2,0} \cap A_{1,0}=\left\{J \in \mathbb{R}^{2}: J_{1}=0, J_{2}=0\right\}$, можно утверждать, что соответствующая конфигурация не является основным состоянием.

2. Пусть $a_{00}=a_{01}=a_{11}=l, a_{10}=m$, где $l, m \in \Phi, l \neq m$.

Пусть $c_{b} \in H_{0}$. Возможны следующие случаи:

a) если $c_{b \downarrow} \in H_{0}$ и $\varphi_{b}\left(c_{b \downarrow}\right)=l$, то $\varphi_{b}\left(c_{b}\right)=l,\left|B_{l}\right|=3,\left|B_{m}\right|=0$, следовательно, $\varphi_{b} \in C_{3,0}$;

б) если $c_{b \downarrow} \in H_{0}$ и $\varphi_{b}\left(c_{b \downarrow}\right)=m$, то $\varphi_{b}\left(c_{b}\right)=l,\left|B_{l}\right|=2,\left|B_{m}\right|=1$, следовательно, $\varphi_{b} \in C_{2,0}$;

в) если $c_{b \downarrow} \in H_{1}$ и $\varphi_{b}\left(c_{b \downarrow}\right)=l$, то $\varphi_{b}\left(c_{b}\right)=m,\left|B_{l}\right|=3,\left|B_{m}\right|=0$, следовательно, $\varphi_{b} \in C_{0,0}$; но, поскольку $A_{3,0} \cap A_{2,0} \cap A_{0,0}=\left\{J \in \mathbb{R}^{2}: J_{1}=0, J_{2}=0\right\}$, соответствующая конфигурация не является основным состоянием.

3. Пусть $a_{00}=a_{10}=a_{11}=l, a_{01}=m$, где $l, m \in \Phi, l \neq m$.

Пусть $c_{b} \in H_{0}$. Возможны следующие случаи:

a) если $c_{b \downarrow} \in H_{0}$ и $\varphi_{b}\left(c_{b \downarrow}\right)=l$, то $\varphi_{b}\left(c_{b}\right)=l,\left|B_{l}\right|=2,\left|B_{m}\right|=1$, следовательно, $\varphi_{b} \in C_{2,0}$;

б) если $c_{b \downarrow} \in H_{1}$ и $\varphi_{b}\left(c_{b \downarrow}\right)=l$, то $\varphi_{b}\left(c_{b}\right)=l,\left|B_{l}\right|=3,\left|B_{m}\right|=0$, следовательно, $\varphi_{b} \in C_{3,0}$.

Пусть $c_{b} \in H_{1}$. Возможен следующий случай: если $c_{b \downarrow} \in H_{0}$ и $\varphi_{b}\left(c_{b \downarrow}\right)=l$, то $\varphi_{b}\left(c_{b}\right)=m,\left|B_{l}\right|=3,\left|B_{m}\right|=0$, следовательно, $\varphi_{b} \in C_{0,0}$; но, поскольку мы имеем $A_{2,0} \cap A_{3,0} \cap A_{0,0}=\left\{J \in \mathbb{R}^{2}: J_{1}=0, J_{2}=0\right\}$, соответствующая конфигурация не является основным состоянием.

4. Пусть $a_{00}=m, a_{01}=a_{10}=a_{11}=l$, где $l, m \in \Phi, l \neq m$.

Пусть $c_{b} \in H_{0}$. Возможен следующий случай: если $c_{b \downarrow} \in H_{0}$ и $\varphi_{b}\left(c_{b \downarrow}\right)=l$, то $\varphi_{b}\left(c_{b}\right)=m,\left|B_{l}\right|=2,\left|B_{m}\right|=1$, следовательно, $\varphi_{b} \in C_{1,0} ;$ но $A_{1,0}=\left\{J \in \mathbb{R}^{2}: J_{1}=\right.$ $\left.0, J_{2}=0\right\}$.

5. Пусть $a_{00}=a_{01}=l, a_{10}=a_{11}=m$, где $l, m \in \Phi, l \neq m$.

Пусть $c_{b} \in H_{0}$. Возможны следующие случаи:

a) если $c_{b \downarrow} \in H_{0}$ и $\varphi_{b}\left(c_{b \downarrow}\right)=l$, то $\varphi_{b}\left(c_{b}\right)=l,\left|B_{l}\right|=3,\left|B_{m}\right|=0$, следовательно, $\varphi_{b} \in C_{3,0}$;

б) если $c_{b \downarrow} \in H_{0}$ и $\varphi_{b}\left(c_{b \downarrow}\right)=m$, то $\varphi_{b}\left(c_{b}\right)=l,\left|B_{l}\right|=2,\left|B_{m}\right|=1$, следовательно, $\varphi_{b} \in C_{2,0}$; 
в) если $c_{b \downarrow} \in H_{1}$ и $\varphi_{b}\left(c_{b \downarrow}\right)=m$, то $\varphi_{b}\left(c_{b}\right)=m,\left|B_{l}\right|=2,\left|B_{m}\right|=1$, следовательно, $\varphi_{b} \in C_{1,0}$; но, поскольку $A_{3,0} \cap A_{2,0} \cap A_{1,0}=\left\{J \in \mathbb{R}^{2}: J_{1}=0, J_{2}=0\right\}$, соответствующая конфигурация не является основным состоянием.

6. Пусть $a_{00}=a_{11}=l, a_{01}=a_{10}=m$, где $l, m \in \Phi, l \neq m$.

Пусть $c_{b} \in H_{0}$. Возможны следующие случаи:

a) если $c_{b \downarrow} \in H_{0}$ и $\varphi_{b}\left(c_{b \downarrow}\right)=l$, то $\varphi_{b}\left(c_{b}\right)=l,\left|B_{l}\right|=2,\left|B_{m}\right|=1$, следовательно, $\varphi_{b} \in C_{2,0}$

б) если $c_{b \downarrow} \in H_{0}$ и $\varphi_{b}\left(c_{b \downarrow}\right)=m$, то $\varphi_{b}\left(c_{b}\right)=l,\left|B_{l}\right|=1,\left|B_{m}\right|=2$, следовательно, $\varphi_{b} \in C_{1,0} ;$ но $A_{2,0} \cap A_{1,0}=\left\{J \in \mathbb{R}^{2}: J_{1}=0, J_{2}=0\right\}$, отсюда получим, что соответствующая конфигурация не является основным состоянием.

7. Пусть $a_{00}=a_{10}=l, a_{01}=a_{11}=m$, где $l, m \in \Phi, l \neq m$. Тогда соответствующие слабо периодические конфигурации имеют следуюший вид:

$$
\varphi(x)=\left\{\begin{array}{ll}
l, & \text { если } x_{\downarrow} \in H_{0}, x \in H_{0}, \\
m, & \text { если } x_{\downarrow} \in H_{0}, x \in H_{1}, \\
l, & \text { если } x_{\downarrow} \in H_{1}, x \in H_{0}, \\
m, & \text { если } x_{\downarrow} \in H_{1}, x \in H_{1}
\end{array}= \begin{cases}l, & \text { если } x \in H_{0}, \\
m, & \text { если } x \in H_{1} .\end{cases}\right.
$$

Отсюда получаем, что слабо периодическая конфигурация (15) является периодической. В работе [6] доказано, что периодическая конфигурация вида $\varphi(x)$ является основным состоянием, следовательно, она также является слабо периодическим основным состоянием.

Рассмотрим случай, когда $a_{i j}$ принимает три разных значения из множества $\Phi$.

Пусть $a_{00}=a_{01}=l, a_{10}=m, a_{11}=n$, где $l, m, n \in \Phi, l \neq m, l \neq n, m \neq n$.

Пусть $c_{b} \in H_{0}$. Возможны следующие случаи:

a) если $c_{b \downarrow} \in H_{0}$ и $\varphi_{b}\left(c_{b \downarrow}\right)=l$, то $\varphi_{b}\left(c_{b}\right)=l,\left|B_{l}\right|=3,\left|B_{m}\right|=0,\left|B_{n}\right|=0$, следовательно, $\varphi_{b} \in C_{3,0}$;

б) если $c_{b \downarrow} \in H_{0}$ и $\varphi_{b}\left(c_{b \downarrow}\right)=m$, то $\varphi_{b}\left(c_{b}\right)=l,\left|B_{l}\right|=2,\left|B_{m}\right|=1,\left|B_{n}\right|=0$, следовательно, $\varphi_{b} \in C_{2,0}$;

в) если $c_{b \downarrow} \in H_{1}$ и $\varphi_{b}\left(c_{b \downarrow}\right)=n$, то $\varphi_{b}\left(c_{b}\right)=m,\left|B_{l}\right|=2,\left|B_{m}\right|=0,\left|B_{n}\right|=1$, следовательно, $\varphi_{b} \in C_{0,1}$; но при этом $A_{3,0} \cap A_{2,0} \cap A_{0,1}=\left\{J \in \mathbb{R}^{2}: J_{1}=0, J_{2}=0\right\}$, отсюда следует, что соответствующая конфигурация не является основным состоянием.

Аналогичным образом анализируя все возможные случаи, можно убедиться, что соответствующие конфигурации не являются основными состояниями. Теорема 1 доказана.

4.2. Доказательство теоремы 2. Докажем сначала первое утверждение теоремы. Рассмотрим конфигурацию $\varphi^{*}(x)$, заданную в $(6)$.

Пусть $c_{b} \in H_{0}$. Возможны следующие случаи:

а) если $c_{b \downarrow} \in H_{0}$ и $\varphi_{b}^{*}\left(c_{b \downarrow}\right)=n$, то $\varphi_{b}^{*}\left(c_{b}\right)=l,\left|B_{l}\right|=0,\left|B_{m}\right|=2,\left|B_{n}\right|=1$, следовательно, $\varphi_{b}^{*} \in C_{0,1}$;

б) если $c_{b \downarrow} \in H_{1}$ и $\varphi_{b}^{*}\left(c_{b \downarrow}\right)=l$, то $\varphi_{b}^{*}\left(c_{b}\right)=n,\left|B_{l}\right|=2,\left|B_{m}\right|=1,\left|B_{n}\right|=0$, следовательно, $\varphi_{b}^{*} \in C_{0,1}$;

в) если $c_{b \downarrow} \in H_{1}$ и $\varphi_{b}^{*}\left(c_{b \downarrow}\right)=m$, то $\varphi_{b}^{*}\left(c_{b}\right)=n,\left|B_{l}\right|=1,\left|B_{m}\right|=2,\left|B_{n}\right|=0$, следовательно, $\varphi_{b}^{*} \in C_{0,1}$. 
Пусть $c_{b} \in H_{1}$. Возможны следующие случаи:

а) если $c_{b \downarrow} \in H_{0}$ и $\varphi_{b}^{*}\left(c_{b \downarrow}\right)=l$, то $\varphi_{b}^{*}\left(c_{b}\right)=m,\left|B_{l}\right|=2,\left|B_{m}\right|=0,\left|B_{n}\right|=1$, следовательно, $\varphi_{b}^{*} \in C_{0,1}$;

б) если $c_{b \downarrow} \in H_{0}$ и $\varphi_{b}^{*}\left(c_{b \downarrow}\right)=n$, то $\varphi_{b}^{*}\left(c_{b}\right)=m,\left|B_{l}\right|=1,\left|B_{m}\right|=0,\left|B_{n}\right|=2$, следовательно, $\varphi_{b}^{*} \in C_{0,1}$;

в) если $c_{b \downarrow} \in H_{1}$ и $\varphi_{b}^{*}\left(c_{b \downarrow}\right)=m$, то $\varphi_{b}^{*}\left(c_{b}\right)=l,\left|B_{l}\right|=0,\left|B_{m}\right|=1,\left|B_{n}\right|=2$, следовательно, $\varphi_{b}^{*} \in C_{0,1}$.

Отсюда следует, что на множестве $A_{0,1}=\left\{J \in \mathbb{R}^{2}: J_{2} \geqslant 0, J_{1}-2 J_{2} \geqslant 0\right\}$ данная слабо периодическая конфигурация $\varphi^{*}(x)$ является слабо периодическим основным состоянием.

Доказательство второго утверждения аналогично доказательству теоремы 1. Теорема 2 доказана.

4.3. Доказательство теоремы 3. Пусть $|A|=1$. Если $a_{i j}=p \in \Phi$ для любых $i, j \in\{0,1\}$, то очевидно, что соответствующие конфигурации являются трансляционно-инвариантными.

Рассмотрим случай, когда $a_{i j}$ принимает два разных значения из множества $\Phi$.

1. Пусть $a_{00}=a_{01}=a_{10}=l, a_{11}=m$, где $l, m \in \Phi, l \neq m$.

Пусть $c_{b} \in H_{0}$. Возможны следующие случаи:

a) если $c_{b \downarrow} \in H_{0}$ и $\varphi_{b}\left(c_{b \downarrow}\right)=l$, то $\varphi_{b}\left(c_{b}\right)=l,\left|B_{l}\right|=4,\left|B_{m}\right|=0$, следовательно, $\varphi_{b} \in C_{4,0}$

б) если $c_{b \downarrow} \in H_{1}$ и $\varphi_{b}\left(c_{b \downarrow}\right)=m$, то $\varphi_{b}\left(c_{b}\right)=l,\left|B_{l}\right|=3,\left|B_{m}\right|=1$, следовательно, $\varphi_{b} \in C_{3,0}$.

Пусть $c_{b} \in H_{1}$. Возможен следующий случай: если $c_{b \downarrow} \in H_{0}$ и $\varphi_{b}\left(c_{b \downarrow}\right)=l$, то $\varphi_{b}\left(c_{b}\right)=l,\left|B_{l}\right|=1,\left|B_{m}\right|=3$, следовательно, $\varphi_{b} \in C_{1,0}$; но при этом мы имеем $A_{4,0} \cap A_{3,0} \cap A_{1,0}=\left\{J \in \mathbb{R}^{2}: J_{1}=0, J_{2}=0\right\}$.

2. Пусть $a_{00}=a_{01}=a_{11}=l, a_{10}=m$, где $l, m \in \Phi, l \neq m$.

Пусть $c_{b} \in H_{0}$. Возможны следующие случаи:

a) если $c_{b \downarrow} \in H_{0}$ и $\varphi_{b}\left(c_{b \downarrow}\right)=l$, то $\varphi_{b}\left(c_{b}\right)=l,\left|B_{l}\right|=4,\left|B_{m}\right|=0$, следовательно, $\varphi_{b} \in C_{4,0}$

б) если $c_{b \downarrow} \in H_{0}$ и $\varphi_{b}\left(c_{b \downarrow}\right)=m$, то $\varphi_{b}\left(c_{b}\right)=l,\left|B_{l}\right|=3,\left|B_{m}\right|=1$, следовательно, $\varphi_{b} \in C_{3,0}$;

в) если $c_{b \downarrow} \in H_{1}$ и $\varphi_{b}\left(c_{b \downarrow}\right)=l$, то $\varphi_{b}\left(c_{b}\right)=m,\left|B_{l}\right|=4,\left|B_{m}\right|=0$, следовательно, $\varphi_{b} \in C_{0,0} ;$ но $A_{4,0} \cap A_{3,0} \cap A_{0,0}=\left\{J \in \mathbb{R}^{2}: J_{1}=0, J_{2}=0\right\}$.

3. Пусть $a_{00}=a_{10}=a_{11}=l, a_{01}=m$, где $l, m \in \Phi, l \neq m$.

Пусть $c_{b} \in H_{0}$. Возможны следующие случаи:

a) если $c_{b \downarrow} \in H_{0}$ и $\varphi_{b}\left(c_{b \downarrow}\right)=l$, то $\varphi_{b}\left(c_{b}\right)=l,\left|B_{l}\right|=3,\left|B_{m}\right|=1$, следовательно, $\varphi_{b} \in C_{3,0}$

б) если $c_{b \downarrow} \in H_{1}$ и $\varphi_{b}\left(c_{b \downarrow}\right)=l$, то $\varphi_{b}\left(c_{b}\right)=l,\left|B_{l}\right|=4,\left|B_{m}\right|=0$, следовательно, $\varphi_{b} \in C_{4,0}$.

Пусть $c_{b} \in H_{1}$. Возможен следующий случай: если $c_{b \downarrow} \in H_{0}$ и $\varphi_{b}\left(c_{b \downarrow}\right)=l$, то $\varphi_{b}\left(c_{b}\right)=m,\left|B_{l}\right|=4,\left|B_{m}\right|=0$, следовательно, $\varphi_{b} \in C_{0,0}$; но при этом мы имеем $A_{3,0} \cap A_{4,0} \cap A_{0,0}=\left\{J \in \mathbb{R}^{2}: J_{1}=0, J_{2}=0\right\}$. 
4. Пусть $a_{00}=m, a_{01}=a_{10}=a_{11}=l$, где $l, m \in \Phi, l \neq m$.

Пусть $c_{b} \in H_{0}$. Возможен следующий случай: если $c_{b \downarrow} \in H_{0}$ и $\varphi_{b}\left(c_{b \downarrow}\right)=l$, то $\varphi_{b}\left(c_{b}\right)=m,\left|B_{l}\right|=2,\left|B_{m}\right|=2$, следовательно, $\varphi_{b} \in C_{2,0}$; но при этом мы имеем $A_{2,0}=\left\{J \in \mathbb{R}^{2}: J_{1}=0, J_{2}=0\right\}$.

5. Пусть $a_{00}=a_{01}=l, a_{10}=a_{11}=m$, где $l, m \in \Phi, l \neq m$.

Пусть $c_{b} \in H_{0}$. Возможны следующие случаи:

a) если $c_{b \downarrow} \in H_{0}$ и $\varphi_{b}\left(c_{b \downarrow}\right)=l$, то $\varphi_{b}\left(c_{b}\right)=l,\left|B_{l}\right|=4,\left|B_{m}\right|=0$, следовательно, $\varphi_{b} \in C_{4,0}$

б) если $c_{b \downarrow} \in H_{0}$ и $\varphi_{b}\left(c_{b \downarrow}\right)=m$, то $\varphi_{b}\left(c_{b}\right)=l,\left|B_{l}\right|=3,\left|B_{m}\right|=1$, следовательно, $\varphi_{b} \in C_{3,0}$;

в) если $c_{b \downarrow} \in H_{1}$ и $\varphi_{b}\left(c_{b \downarrow}\right)=m$, то $\varphi_{b}\left(c_{b}\right)=m,\left|B_{l}\right|=3,\left|B_{m}\right|=1$, следовательно, $\varphi_{b} \in C_{1,0} ;$ но $A_{4,0} \cap A_{3,0} \cap A_{1,0}=\left\{J \in \mathbb{R}^{2}: J_{1}=0, J_{2}=0\right\}$.

6. Пусть $a_{00}=a_{11}=l, a_{01}=a_{10}=m$, где $l, m \in \Phi, l \neq m$.

Пусть $c_{b} \in H_{0}$. Возможны следующие случаи:

a) если $c_{b \downarrow} \in H_{0}$ и $\varphi_{b}\left(c_{b \downarrow}\right)=l$, то $\varphi_{b}\left(c_{b}\right)=l,\left|B_{l}\right|=3,\left|B_{m}\right|=1$, следовательно, $\varphi_{b} \in C_{3,0}$.

б) если $c_{b \downarrow} \in H_{0}$ и $\varphi_{b}\left(c_{b \downarrow}\right)=m$, то $\varphi_{b}\left(c_{b}\right)=l,\left|B_{l}\right|=2,\left|B_{m}\right|=2$, следовательно, $\varphi_{b} \in C_{2,0}$; но $A_{3,0} \cap A_{2,0}=\left\{J \in \mathbb{R}^{2}: J_{1}=0, J_{2}=0\right\}$, поэтому можно утверждать, что соответствующие конфигурации не являются основными состояниями.

7. Пусть $a_{00}=a_{10}=l, a_{01}=a_{11}=m$, где $l, m \in \Phi, l \neq m$. Тогда соответствующая слабо периодическая конфигурация имеет вид (15).

Пусть $c_{b} \in H_{0}$. Возможны следующие случаи:

a) если $c_{b \downarrow} \in H_{0}$ и $\varphi_{b}\left(c_{b \downarrow}\right)=l$, то $\varphi_{b}\left(c_{b}\right)=l,\left|B_{l}\right|=3,\left|B_{m}\right|=1$, следовательно, $\varphi_{b} \in C_{3,0}$

б) если $c_{b \downarrow} \in H_{1}$ и $\varphi_{b}\left(c_{b \downarrow}\right)=m$, то $\varphi_{b}\left(c_{b}\right)=l,\left|B_{l}\right|=3,\left|B_{m}\right|=1$, следовательно, $\varphi_{b} \in C_{3,0}$.

Пусть $c_{b} \in H_{1}$. Возможны следующие случаи:

a) если $c_{b \downarrow} \in H_{0}$ и $\varphi_{b}\left(c_{b \downarrow}\right)=l$, то $\varphi_{b}\left(c_{b}\right)=m,\left|B_{l}\right|=1,\left|B_{m}\right|=3$, следовательно, $\varphi_{b} \in C_{3,0}$;

б) если $c_{b \downarrow} \in H_{1}$ и $\varphi_{b}\left(c_{b \downarrow}\right)=m$, то $\varphi_{b}\left(c_{b}\right)=m,\left|B_{l}\right|=1,\left|B_{m}\right|=3$, следовательно, $\varphi_{b} \in C_{3,0}$; но $A_{3,0}=\left\{J \in \mathbb{R}^{2}: J_{1} \leqslant 0,-J_{1} / 6 \leqslant J_{2} \leqslant-J_{1} / 4\right\}$, отсюда получим, что слабо периодическая конфигурация $\varphi(x)$ является периодической; периодическая конфигурация $\varphi(x)$ является основным состоянием, следовательно, она является также слабо периодическим основным состоянием.

Рассмотрим случай, когда $a_{i j}$ принимает три разных значения из множества $\Phi$.

1. Пусть $a_{00}=a_{01}=l, a_{10}=m, a_{11}=n$, где $l, m, n \in \Phi, l \neq m, l \neq n, m \neq n$.

Пусть $c_{b} \in H_{0}$. Возможны следующие случаи:

а) если $c_{b \downarrow} \in H_{0}$ и $\varphi_{b}\left(c_{b \downarrow}\right)=l$, то $\varphi_{b}\left(c_{b}\right)=l,\left|B_{l}\right|=4,\left|B_{m}\right|=0,\left|B_{n}\right|=0$, следовательно, $\varphi_{b} \in C_{4,0}$;

б) если $c_{b \downarrow} \in H_{0}$ и $\varphi_{b}\left(c_{b \downarrow}\right)=m$, то $\varphi_{b}\left(c_{b}\right)=l,\left|B_{l}\right|=3,\left|B_{m}\right|=1,\left|B_{n}\right|=0$, следовательно, $\varphi_{b} \in C_{3,0}$;

в) если $c_{b \downarrow} \in H_{1}$ и $\varphi_{b}\left(c_{b \downarrow}\right)=n$, то $\varphi_{b}\left(c_{b}\right)=m,\left|B_{l}\right|=3,\left|B_{m}\right|=0,\left|B_{n}\right|=1$, следовательно, $\varphi_{b} \in C_{0,1} ;$ но $A_{4,0} \cap A_{3,0} \cap A_{0,1}=\left\{J \in \mathbb{R}^{2}: J_{1}=0, J_{2}=0\right\}$. 
2. Пусть $a_{00}=a_{10}=l, a_{01}=m, a_{11}=n$, где $l, m, n \in \Phi, l \neq m, l \neq n, m \neq n$.

Пусть $c_{b} \in H_{0}$. Возможны следующие случаи:

а) если $c_{b \downarrow} \in H_{0}$ и $\varphi_{b}\left(c_{b \downarrow}\right)=l$, то $\varphi_{b}\left(c_{b}\right)=l,\left|B_{l}\right|=3,\left|B_{m}\right|=1,\left|B_{n}\right|=0$, следовательно, $\varphi_{b} \in C_{3,0}$;

б) если $c_{b \downarrow} \in H_{1}$ и $\varphi_{b}\left(c_{b \downarrow}\right)=n$, то $\varphi_{b}\left(c_{b}\right)=l,\left|B_{l}\right|=3,\left|B_{m}\right|=0,\left|B_{n}\right|=1$, следовательно, $\varphi_{b} \in C_{3,0}$.

Пусть $c_{b} \in H_{1}$. Возможен следующий случай: если $c_{b \downarrow} \in H_{0}$ и $\varphi_{b}\left(c_{b \downarrow}\right)=l$, то $\varphi_{b}\left(c_{b}\right)=m,\left|B_{l}\right|=1,\left|B_{m}\right|=0,\left|B_{n}\right|=3$, следовательно, $\varphi_{b} \in C_{0,1}$; но при этом мы имеем $A_{3,0} \cap A_{0,1}=\left\{J \in \mathbb{R}^{2}: J_{1}=0, J_{2}=0\right\}$.

3. Пусть $a_{00}=a_{11}=l, a_{01}=m, a_{10}=n$, где $l, m, n \in \Phi, l \neq m, l \neq n, m \neq n$.

Пусть $c_{b} \in H_{0}$. Возможны следующие случаи:

а) если $c_{b \downarrow} \in H_{0}$ и $\varphi_{b}\left(c_{b \downarrow}\right)=l$, то $\varphi_{b}\left(c_{b}\right)=l,\left|B_{l}\right|=3,\left|B_{m}\right|=1,\left|B_{n}\right|=0$, следовательно, $\varphi_{b} \in C_{3,0}$;

б) если $c_{b \downarrow} \in H_{0}$ и $\varphi_{b}\left(c_{b \downarrow}\right)=n$, то $\varphi_{b}\left(c_{b}\right)=l,\left|B_{l}\right|=2,\left|B_{m}\right|=1,\left|B_{n}\right|=1$, следовательно, $\varphi_{b} \in C_{2,1}$;

в) если $c_{b \downarrow} \in H_{1}$ и $\varphi_{b}\left(c_{b \downarrow}\right)=l$, то $\varphi_{b}\left(c_{b}\right)=n,\left|B_{l}\right|=4,\left|B_{m}\right|=0,\left|B_{n}\right|=0$, следовательно, $\varphi_{b} \in C_{0,0}$; но $A_{3,0} \cap A_{2,1} \cap A_{0,0}=\left\{J \in \mathbb{R}^{2}: J_{1}=0, J_{2}=0\right\}$.

4. Пусть $a_{00}=m, a_{01}=a_{10}=l, a_{11}=n$, где $l, m, n \in \Phi, l \neq m, l \neq n, m \neq n$.

Пусть $c_{b} \in H_{0}$. Возможен следующий случай: если $c_{b \downarrow} \in H_{0}$ и $\varphi_{b}\left(c_{b \downarrow}\right)=l$, то $\varphi_{b}\left(c_{b}\right)=m,\left|B_{l}\right|=2,\left|B_{m}\right|=2,\left|B_{n}\right|=0$, следовательно, $\varphi_{b} \in C_{2,0}$; но при этом мы имеем $A_{2,0}=\left\{J \in \mathbb{R}^{2}: J_{1}=0, J_{2}=0\right\}$.

5. Пусть $a_{00}=m, a_{01}=a_{11}=l, a_{10}=n$, где $l, m, n \in \Phi, l \neq m, l \neq n, m \neq n$.

Пусть $c_{b} \in H_{0}$. Возможны следующие случаи:

а) если $c_{b \downarrow} \in H_{0}$ и $\varphi_{b}\left(c_{b \downarrow}\right)=m$, то $\varphi_{b}\left(c_{b}\right)=m,\left|B_{l}\right|=1,\left|B_{m}\right|=3,\left|B_{n}\right|=0$, следовательно, $\varphi_{b} \in C_{3,0}$;

б) если $c_{b \downarrow} \in H_{0}$ и $\varphi_{b}\left(c_{b \downarrow}\right)=n$, то $\varphi_{b}\left(c_{b}\right)=m,\left|B_{l}\right|=1,\left|B_{m}\right|=2,\left|B_{n}\right|=1$, следовательно, $\varphi_{b} \in C_{2,1}$;

в) если $c_{b \downarrow} \in H_{1}$ и $\varphi_{b}\left(c_{b \downarrow}\right)=l$, то $\varphi_{b}\left(c_{b}\right)=n,\left|B_{l}\right|=1,\left|B_{m}\right|=3,\left|B_{n}\right|=0$, следовательно, $\varphi_{b} \in C_{0,1}$; но мы имеем $A_{3,0} \cap A_{2,1} \cap A_{0,1}=\left\{J \in \mathbb{R}^{2}: J_{1}=0, J_{2}=0\right\}$.

6. Пусть $a_{00}=m, a_{01}=n, a_{10}=a_{11}=l$, где $l, m, n \in \Phi, l \neq m, l \neq n, m \neq n$.

Пусть $c_{b} \in H_{0}$. Возможны следующие случаи:

а) если $c_{b \downarrow} \in H_{0}$ и $\varphi_{b}\left(c_{b \downarrow}\right)=l$, то $\varphi_{b}\left(c_{b}\right)=m,\left|B_{l}\right|=1,\left|B_{m}\right|=2,\left|B_{n}\right|=1$, следовательно, $\varphi_{b} \in C_{2,1}$;

б) если $c_{b \downarrow} \in H_{0}$ и $\varphi_{b}\left(c_{b \downarrow}\right)=m$, то $\varphi_{b}\left(c_{b}\right)=m,\left|B_{l}\right|=0,\left|B_{m}\right|=3,\left|B_{n}\right|=1$, следовательно, $\varphi_{b} \in C_{3,0}$;

в) если $c_{b \downarrow} \in H_{1}$ и $\varphi_{b}\left(c_{b \downarrow}\right)=l$, то $\varphi_{b}\left(c_{b}\right)=l,\left|B_{l}\right|=1,\left|B_{m}\right|=3,\left|B_{n}\right|=0$, следовательно, $\varphi_{b} \in C_{1,0}$; но $A_{2,1} \cap A_{3,0} \cap A_{1,0}=\left\{J \in \mathbb{R}^{2}: J_{1}=0, J_{2}=0\right\}$; на основании рассуждений, приведенных выше, можно утверждать, что соответствующие конфигурации не являются основными состояниями.

Случай $|A|=2$ доказывается аналогично. Теорема 3 доказана.

4.4. Доказательство теоремы 4. Докажем первое утверждение теоремы.

Пусть $c_{b} \in H_{0}$. Возможны следующие случаи:

а) если $c_{b \downarrow} \in H_{0}$ и $\varphi_{b}^{*}\left(c_{b \downarrow}\right)=n$, то $\varphi_{b}^{*}\left(c_{b}\right)=l,\left|B_{l}\right|=0,\left|B_{m}\right|=3,\left|B_{n}\right|=1$, следовательно, $\varphi_{b}^{*} \in C_{0,1}$; 
б) если $c_{b \downarrow} \in H_{1}$ и $\varphi_{b}^{*}\left(c_{b \downarrow}\right)=l$, то $\varphi_{b}^{*}\left(c_{b}\right)=n,\left|B_{l}\right|=2,\left|B_{m}\right|=2,\left|B_{n}\right|=0$, следовательно, $\varphi_{b}^{*} \in C_{0,2}$;

в) если $c_{b \downarrow} \in H_{1}$ и $\varphi_{b}^{*}\left(c_{b \downarrow}\right)=m$, то $\varphi_{b}^{*}\left(c_{b}\right)=n,\left|B_{l}\right|=1,\left|B_{m}\right|=3,\left|B_{n}\right|=0$, следовательно, $\varphi_{b}^{*} \in C_{0,1}$.

Пусть $c_{b} \in H_{1}$. Возможны следующие случаи:

а) если $c_{b \downarrow} \in H_{0}$ и $\varphi_{b}^{*}\left(c_{b \downarrow}\right)=l$, то $\varphi_{b}^{*}\left(c_{b}\right)=m,\left|B_{l}\right|=2,\left|B_{m}\right|=0,\left|B_{n}\right|=2$, следовательно, $\varphi_{b}^{*} \in C_{0,2}$;

б) если $c_{b \downarrow} \in H_{0}$ и $\varphi_{b}^{*}\left(c_{b \downarrow}\right)=n$, то $\varphi_{b}^{*}\left(c_{b}\right)=m,\left|B_{l}\right|=1,\left|B_{m}\right|=0,\left|B_{n}\right|=3$, следовательно, $\varphi_{b}^{*} \in C_{0,1}$;

в) если $c_{b \downarrow} \in H_{1}$ и $\varphi_{b}^{*}\left(c_{b \downarrow}\right)=m$, то $\varphi_{b}^{*}\left(c_{b}\right)=l,\left|B_{l}\right|=0,\left|B_{m}\right|=1,\left|B_{n}\right|=3$, следовательно, $\varphi_{b}^{*} \in C_{0,1}$.

Отсюда получим, что на множестве $A_{0,1} \cap A_{0,2}=A_{0,1}=\left\{J \in \mathbb{R}^{2}: J_{1} \geqslant 0, J_{2}=0\right\}$ данная конфигурация $\varphi^{*}(x)$ является слабо периодическим основным состоянием.

Доказательство второго утверждения теоремы аналогично доказательству теоремы 3. Теорема 4 доказана.

4.5. Доказательство теоремы 5. Пусть $c_{b} \in H_{0}$. Возможны следующие случаи:

a) если $c_{b \downarrow} \in H_{0}$ и $\varphi_{b}^{*}\left(c_{b \downarrow}\right)=n$, то $\varphi_{b}^{*}\left(c_{b}\right)=l,\left|B_{l}\right|=0,\left|B_{m}\right|=k,\left|B_{n}\right|=1$, следовательно, $\varphi_{b}^{*} \in C_{0,1}$;

б) если $c_{b \downarrow} \in H_{1}$ и $\varphi_{b}^{*}\left(c_{b \downarrow}\right)=l$, то $\varphi_{b}^{*}\left(c_{b}\right)=n,\left|B_{l}\right|=2,\left|B_{m}\right|=k-1,\left|B_{n}\right|=0$, следовательно, $\varphi_{b}^{*} \in C_{0,2}$;

в) если $c_{b \downarrow} \in H_{1}$ и $\varphi_{b}^{*}\left(c_{b \downarrow}\right)=m$, то $\varphi_{b}^{*}\left(c_{b}\right)=n,\left|B_{l}\right|=1,\left|B_{m}\right|=k,\left|B_{n}\right|=0$, следовательно, $\varphi_{b}^{*} \in C_{0,1}$.

Пусть $c_{b} \in H_{1}$. Возможны следующие случаи:

а) если $c_{b \downarrow} \in H_{0}$ и $\varphi_{b}^{*}\left(c_{b \downarrow}\right)=l$, то $\varphi_{b}^{*}\left(c_{b}\right)=m,\left|B_{l}\right|=2,\left|B_{m}\right|=0,\left|B_{n}\right|=k-1$, следовательно, $\varphi_{b}^{*} \in C_{0,2}$;

б) если $c_{b \downarrow} \in H_{0}$ и $\varphi_{b}^{*}\left(c_{b \downarrow}\right)=n$, то $\varphi_{b}^{*}\left(c_{b}\right)=m,\left|B_{l}\right|=1,\left|B_{m}\right|=0,\left|B_{n}\right|=k$, следовательно, $\varphi_{b}^{*} \in C_{0,1}$;

в) если $c_{b \downarrow} \in H_{1}$ и $\varphi_{b}^{*}\left(c_{b \downarrow}\right)=m$, то $\varphi_{b}^{*}\left(c_{b}\right)=l,\left|B_{l}\right|=0,\left|B_{m}\right|=1,\left|B_{n}\right|=k$, следовательно, $\varphi_{b}^{*} \in C_{0,1}$.

Таким образом, на множестве $A_{0,1} \cap A_{0,2}=\left\{J \in \mathbb{R}^{2}: J_{1} \geqslant 0, J_{2}=0\right\}$ данная конфигурация $\varphi^{*}(x)$ является слабо периодическим основным состоянием. Теорема 5 доказана.

4.6. Доказательство теоремы 7. Докажем первое утверждение теоремы. Для инвариантного множества $I_{1}$ доказательство очевидно.

Докажем второе утверждение теоремы для инвариантного множества $I_{2}$. Система уравнений (10) при $k=|A|=2$ на инвариантном множестве $I_{2}$ имеет следующий вид:

$$
z_{11}=\left(\frac{(\theta+q-2) z_{21}+1}{(q-1) z_{21}+\theta}\right)^{2}, \quad z_{21}=\left(\frac{(\theta+q-2) z_{11}+1}{(q-1) z_{11}+\theta}\right)\left(\frac{(\theta+q-2) z_{21}+1}{(q-1) z_{21}+\theta}\right) .
$$

Подставляя первое уравнение во второе, получим равенство

$$
z_{21}=\left(\frac{(\theta+q-2) z_{11}+1}{(q-1) z_{11}+\theta}\right) \sqrt{z_{11}}
$$


подставим его в первое уравнение и положим $z=\sqrt{z_{11}}$. В результате получим уравнение

$$
(q-1)(\theta+q-2) z^{4}+\left(\theta(q-1)-(\theta+q-2)^{2}\right) z^{3}+\left(\theta^{2}-(\theta+q-2)\right) z-\theta=0,
$$

левая часть которого делится на $z-1$. Производя это деление, получим

$$
\left(q^{2}+\theta q-3 q-\theta+2\right) z^{3}+\left(q+2 \theta-\theta^{2}-2\right) z^{2}+\left(q+2 \theta-\theta^{2}-2\right) z+\theta=0 .
$$

Это уравнение является кубическим относительно $z$, с другой стороны, оно является квадратным уравнением относительно $\theta$. Найдем решения этого квадратного уравнения:

$$
\theta_{1}=-\frac{(q-2) z}{z+1}, \quad \theta_{2}=\frac{(q-1) z^{2}+z+1}{z} .
$$

Неравенство $\theta=-(q-2) z /(z+1)<0$ при $q>2, z>0$ невозможно.

Для $\theta=\theta_{2}$ имеем

$$
(q-1) z^{2}+(1-\theta) z+1=0 .
$$

Если $\theta^{2}-2 \theta+5-4 q=0$, что эквивалентно $\theta=1 \pm 2 \sqrt{q-1}$, то уравнение (16) имеет единственное решение, так как параметр $\theta>0$ может принимать только значение $\theta=1+2 \sqrt{q-1}$. Единственное решение уравнения (16) имеет вид

$$
z=\frac{\sqrt{\theta-1}}{2(q-1)}=\frac{1}{\sqrt{q-1}}
$$

и положительно. Если $\theta^{2}-2 \theta-7>0$, что эквивалентно

$$
\theta \in(-\infty, 1-2 \sqrt{q-1}) \cup(1+2 \sqrt{q-1},+\infty),
$$

то уравнение (16) имеет два решения, так как параметр $\theta>0$ может принимать только значения из интервала $(1+2 \sqrt{q-1},+\infty)$. Легко проверить, что для таких $\theta$ оба решения уравнения (16) являются положительными.

Доказательство второго утверждения в случае $|A|=1$ аналогично. Теорема 7 доказана.

4.7. Доказательство леммы 3. Уравнение (13) можно переписать как

$$
\frac{\theta+q-2}{q-1} \frac{\frac{(\theta+q-2) z^{2 k-1}+z^{k-1}}{(q-1) z^{k}+\theta}+1}{\frac{(\theta+q-2) z^{2 k-1}+z^{k-1}}{(q-1) z^{k}+\theta}+\theta}=z
$$

или как

$$
\left((\theta+q-2)^{2}-z(q-1)(\theta+q-2)\right) z^{2 k}+\left((\theta+q-2)-\theta z^{2}(q-1)\right) z^{k}+\theta z-\theta^{2} z^{2}=0 .
$$

Отсюда находим $z^{k}$ как корни квадратного уравнения: для $z^{k}=-\theta z /(\theta+q-2)$ неравенство $z^{k}<0$ при $\theta>0, q \geqslant 2$ невозможно; для другого корня квадратного уравнения имеем

$$
z^{k}=\frac{\theta z-1}{(1-q) z+\theta+q-2}, \quad z=\frac{(\theta+q-2) z^{k}+1}{(q-1) z^{k}+\theta}=f\left(z^{k}\right) .
$$

Лемма 3 доказана. 
4.8. Доказательство теоремы 8. Уравнение

$$
z=f\left(z^{k}\right)=(\theta+q-2) z^{k}+1(q-1) z^{k}+\theta
$$

перепишем как уравнение

$$
(q-1) z^{k+1}-(\theta+q-2) z^{k}+\theta z-1=0
$$

одним из решений которого является $z=1$. Тогда, разделив левую часть уравнения (17) на $z-1$, получим следующее уравнение:

$$
(q-1) z^{k}-(\theta-1)\left(z^{k-1}+z^{k-2}+\cdots+z\right)+1=0 .
$$

Рассмотрим функцию $g(z)=(q-1) z^{k}-(\theta-1)\left(z^{k-1}+z^{k-2}+\cdots+z\right)+1$. Легко проверить, что $g(0)=1$. Существует значение $\theta_{\text {cr }}>1$ такое, что при $\theta>\theta_{\text {сr }}$ множество $\{z: g(z)<0\}$ непустое, но при этом $g(z)>0$ для достаточно больших $z$, т. е. функция $g(z)$ имеет два нуля. Если $\theta=\theta_{\text {cr }}$, то функция $g(z)$ имеет один ноль. Теорема 8 доказана.

Благодарности. Автор выражает свою глубокую признательность профессору У.А. Розикову за постановку задачи и полезные советы по работе.

\section{Список литературы}

[1] Я. Г. Синай, Теория фазовых переходов. Строгие результаты, Наука, М., 1980.

[2] Р.А. Минлос, Введение в математическую статистическую физику, МЦНМО, М., 2002.

[3] У. А. Розиков, М. М. Рахматуллаев, ТМФ, 160:3 (2009), 507-516.

[4] M. M. Rahmatullaev, Appl. Math. Inf. Sci., 4:2 (2010), 237-251.

[5] У. А. Розиков, М. М. Рахматуллаев, ТМФ, 156:2 (2008), 292-302.

[6] Г. И. Ботиров, У. А. Розиков, ТМФ, 153:1 (2007), 86-97.

[7] Н. Н. Ганиходжаев, У. А. Розиков, ТМФ, 111:1 (1997), 109-117.

[8] У. А. Розиков, ТМФ, 112:1 (1997), 170-175.

[9] П. М. Блехер, Н. Н. Ганиходжаев, ТВП, 35:2 (1990), 220-230.

Поступила в редакцию 11.03.2013 